\title{
The Use of Auditory Brainstem Response (ABR) as Indicator of Neural Integrity in Newborn and Infants Submitted to High Complexity Cardiac Surgery
}

\author{
Elaine Cristina Ramos, Henrique Furlan Pauna*, Jyrson Guilherme Klamt and Miguel Angelo Hyppolito \\ Department of Ophthalmology, Otorhinolaryngology and Head and Neck Surgery, University of São Paulo (FMRP-USP), Brazil
}

*Corresponding author: Henrique Furlan Pauna, Department of Ophthalmology, Otorhinolaryngology and Head and Neck Surgery. Ribeirão Preto Medical School, University of São Paulo (FMRP-USP), Ribeirão Preto, São Paulo, Brazil

\begin{abstract}
Introduction: Neurological injury is an important cause of morbidity and post-operatory mortality in high complexity cardiac surgery in children. The auditory brainstem response (ABR) evaluates specific areas of the brainstem. It provides useful information about the functional state of the brain during important cardiovascular procedure.

Objective: To assess the progression in ABR waves as an indicator of neural integrity during controlled brain hypoxia-ischemia among children undergoing to cardiac surgery with cardiopulmonary bypass (CPB).

Methods: Nine pediatric patients aged 0 to 2 years-old were included. The electrophysiological assessment - by means of ABR - was performed at the operating room, during the following established steps of the cardiac surgery: general anesthesia ( $\mathrm{t} 0$ ), $\mathrm{CPB}$ and hypothermia ( $\mathrm{t} 1$ ), and at the end of surgery ( $\mathrm{t} 2$ ).

Results: Mean age of children was 10 months. Out of the 9 children, 3 were female and 6 were male. We observed increased latency for waves I, III, and V. We also observed an increasing interpeak latency (I-III, I-V, and III-V) for both ears during CPB and hypothermia (t1). Despite the increase in both average latencies and interpeak latencies, the values were not statistically significant.

Conclusion: No significant changes were found in the mean latencies and interpeak latencies of ABR during CPB and hypothermia in complex cardiac surgeries included in the present study. ABR seems to be adequate to monitor patients during general anesthesia.
\end{abstract}

Keywords: Auditory brainstem response; Cardiac surgery; auditory pathway; General anesthesia.

\section{Introduction}

Neurological injury is an important cause of morbidity and post-operatory mortality in cardiac surgery. Brain ischemia, embolization and inflammation have been suggested as potential mechanisms of the aforementioned injury [1]. The decline in mortality rates due to congenital cardiac disease has been followed by an increase of sequel development and morbidities with possible causes associated with the brain injury in new-born and children submitted to open chest heart surgery. Such injury has multifactorial causes which include a complex interaction among the pre, intra, and postoperative events. There is an increased risk for brain injury risk of brain injury as a result of adverse neurological development associated with the prolonged exposure to deep hypothermia circulatory arrest [2]. The neurological injury still occurs in children as a consequence of cardiac surgery with extracorporeal circulation, despite the progress achieved in reducing the mortality in the last decades, which is currently below $1 \%$. American statistics show that 40 to $50 \%$ of children aged between 4 and 5 who are submitted to complex cardiac surgeries during neonatal period or new-borns aged less than six months show disability in neurocognitive development, which includes cognitive, motor, and emotional deficit, fine motor disability, language, speech, motor visual organization deficit, and attention deficit and hyperactivity [3-6]. Auditory brainstem response (ABR) 
is a short latency potential which generates a series of waves classified I to VII that emerges in the first 10 milliseconds after the presentation of the auditory stimulus. These waves are generated by sequential activation of structures from the cochlea and caught by electrodes located in the skin.

Recent studies suggest that the location of the following waves represent: Wave I - distal portion of the auditory nerve, wave II proximal portion of the auditory nerve, wave III - cochlear nucleus, wave IV - superior olivary complex, wave V - lateral lemniscus, waves VI and VII - inferior colliculus. The ABR is an objective, noninvasive method that allows the neurophysiological analysis of the ear canal, from the inner ear to the high brainstem [7]. ABR evaluates specific areas of the brainstem. It provides useful information about the functional state of the brain during important cardiovascular procedure and is adequate to monitor patients during general anaesthesia and critical care [1]. It is not currently determined how intraoperative events associated or correlated to cerebral hypoxia-ischemia can affect the evoked potentials momentarily or definitively. There is evidence that anaesthetic drugs to be used do not modify the evoked potentials and, therefore, significant latency, wavelength, interwave latency alteration and its morphologies may be indicative of acute neurological dysfunction and predictive of long term behavioral cognitive sequels $[8,9]$. Therefore, our objective is to determine whether changes in the waves of ABR may work as an indicator of neural integrity during brain hypoxia-ischemia in children undergoing cardiac surgery with cardiopulmonary bypass (CPB).

\section{Materials and Methods}

Nineteen paediatric patients (under 18 years of age) submitted to high complexity cardiac surgery (with cardio pulmonary bypass and hypothermia) in an University Hospital were selected for the present study. The cardiac surgeries were performed from April 2016 to November 2016. All procedures contributing to the present study comply with the ethical standards of the Institutional Guidelines on human experimentation (CEP 16637/2015) and with the Helsinki Declaration of 1975, as revised in 2008.

\section{Exclusion criteria}

Clinically evident injury or neurological abnormalities, clinically evident middle ear conditions (such as acute otitis media, serous otitis media, or chronic otitis media), patients with longterm sedation and oro-tracheal intubation prior to surgery, patients receiving vasoactive drugs except for milrinone or prostaglandin E2 at low doses, preterm patients (gestational age shorter than 34 weeks), severe preoperative hypoxia (oxygen saturation lower than 70\%), Apgar scores lower than 3 after five and ten minutes after birth, perinatal central nervous system (CNS) aggressions such as birth hypoxia and cardiac arrest, critical general condition with multiple organ dysfunctions, and absence of identifiable and reproducible waves in the first register that were considered a basic register for the analysis of the others.
After applying our rigorous exclusion criteria, our final paediatric group included nine patients ( 3 females and 6 males), mean age of 10 months (range, 28 days to 22 months). Two of the children had Down syndrome associated, with no other pathology otherwise the cardiac impairment.

\section{Electrophysiological assessment}

Electrophysiological evaluation was conducted in the operating room with a proper electrical grounding that allowed the tracing to be obtained with no electrical interference. The following steps of the cardiac surgery were considered: general anaesthesia (onset of surgery, $\mathrm{t} 0$ ), extracorporeal bypass and hypothermia ( $\mathrm{t} 1$ ), and by the end of the surgery ( $\mathrm{t} 2$ ), by means of ABR testing. Subcutaneous needle electrodes were placed and affixed when the patient was already under general anaesthesia. The electrodes were placed in the following order: one positive electrode placed on the cranial vertex, two electrodes placed on the mastoid, one being placed on the right mastoid and another on the left and one reference (ground wire) placed on the front (between the eyes). For data acquisition and analysis, Nicolet Endeavour CR $^{\mathrm{TM}}$ equipment (VIASYS Neuro Care, Madison, WI, USA) was used, which was connected to a laptop, insertion earphones, and four needle electrodes. The register of ABR was considered at the aforementioned steps: general anaesthesia ( $\mathrm{t} 0$ ); as base line and or reference to comparison of subsequent registers), CPB and hypothermia ( $\mathrm{t} 1$ ), and at the end of the surgery (t2). The first trace was used as an initial pattern of comparison for the subsequent steps of analysis. The induction of general anaesthesia was performed with low doses of propofol (concentration of $1.5 \mathrm{mg} / \mathrm{kg}$ ), and it was maintained in a scheme with infusion of propofol up to $3.0 \mathrm{mg} / \mathrm{kg}$ ) [8]. Additionally, the temperature was controlled for each patient during the surgery, and the level of temperature was determined by the anesthetist for each case.

The sound stimulus was presented randomly to the right and left ears. The applied stimulus was an alternating "click" type, with an average of 11.4 stimuli per second, for 0.1 milliseconds, between $2,000 \mathrm{~Hz}$ and $4,000 \mathrm{~Hz}$, and using the fixed threshold of $90 \mathrm{dBHL}$. The signal obtained by the electrodes was filtered with high and low pass filters of $150 \mathrm{~Hz}$ and 2,000 Hz, respectively. There was replication of each wave obtained. In ABR, the amplified and filtered signal was calculated with an average of 1,000 scans of the evoked response records within 10 milliseconds. The final result was provided as electric potential waves.

The primary parameter of analysis was the shift of the ABR latencies between the first register (at t0) and the end of the surgery, cardiopulmonary bypass and hypothermia ( $\mathrm{t} 1$ and $\mathrm{t} 2$ ). The latency scores of waves I, III, and V, as well as the interpeak scores of waves I-III, III-V, and I-V were analysed by two independent experienced professionals. If no consensus was achieved between the two judges, a third judge would make a new analysis and would have the final decision. 


\section{Statistical analysis}

The Mann-Whitney U test was used, along with qualitative analysis of the data, and results were considered statistically significant when $\mathrm{p}$ value was $<0.05$.

\section{Results}

The epidemiological data is present in Table 1. We obtained the clear waves I, III, and V at baseline evaluation and mean values from nine left ears and eight right ears are presented in Table 2. No differences between right and left ears at the baseline evaluation or during the surgery were found ( $p>0.05)$. As for the latencies of the waves I, III, and V and their interpeak of the right and left ear, it was observed small changes in values compared to the onset with CPB and hypothermia, as well as compared to the beginning and the end of the surgery, but the results were not statistically significant ( $p$ > 0.05 , Figures 1-4). The absence of waves I, III, and V in the left ear during cardiopulmonary by-pass and hypothermia was observed in just one subject. As for the subject's right ear, the absence of waves I, III, and V was observed in all moments of the cardiac surgery. We found no changes on ABR latencies and wave amplitudes during general anaesthesia.

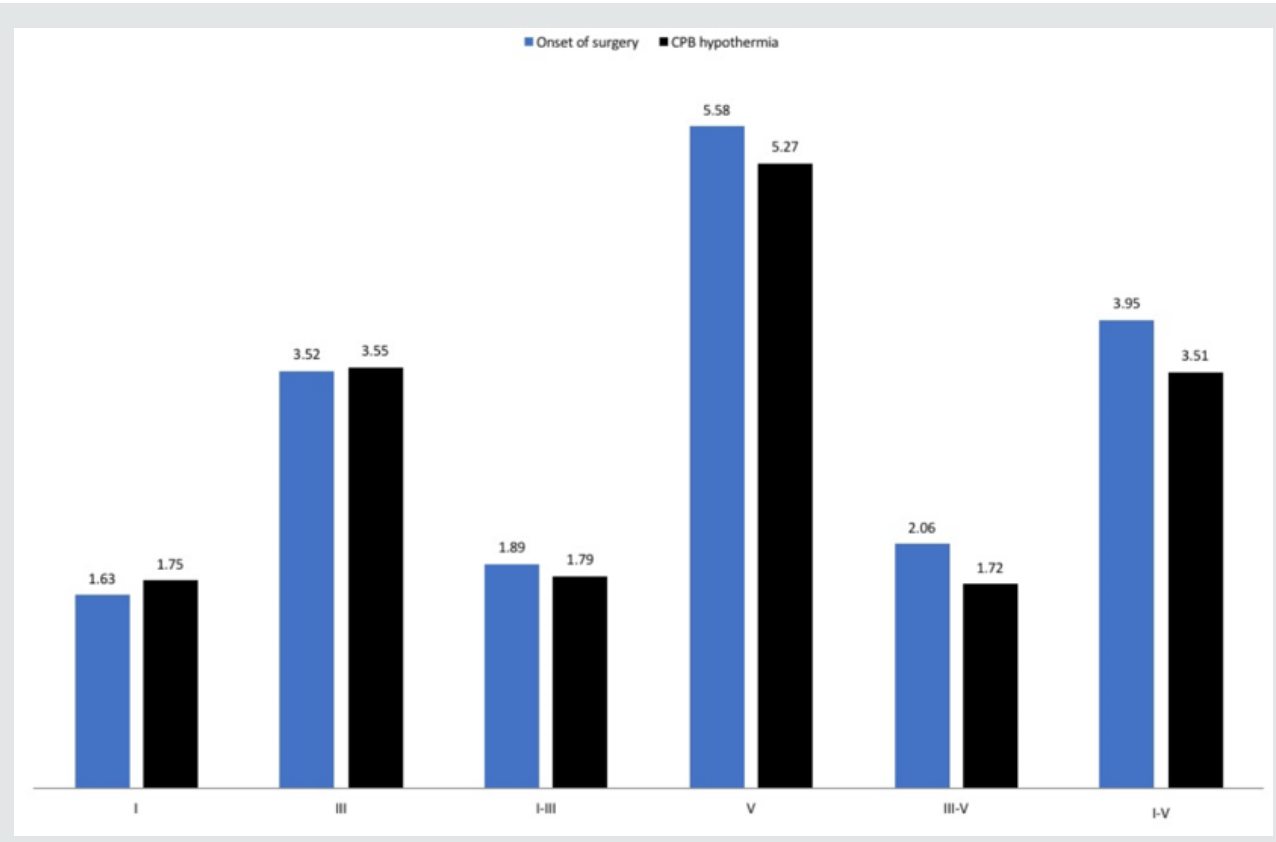

Figure 1.

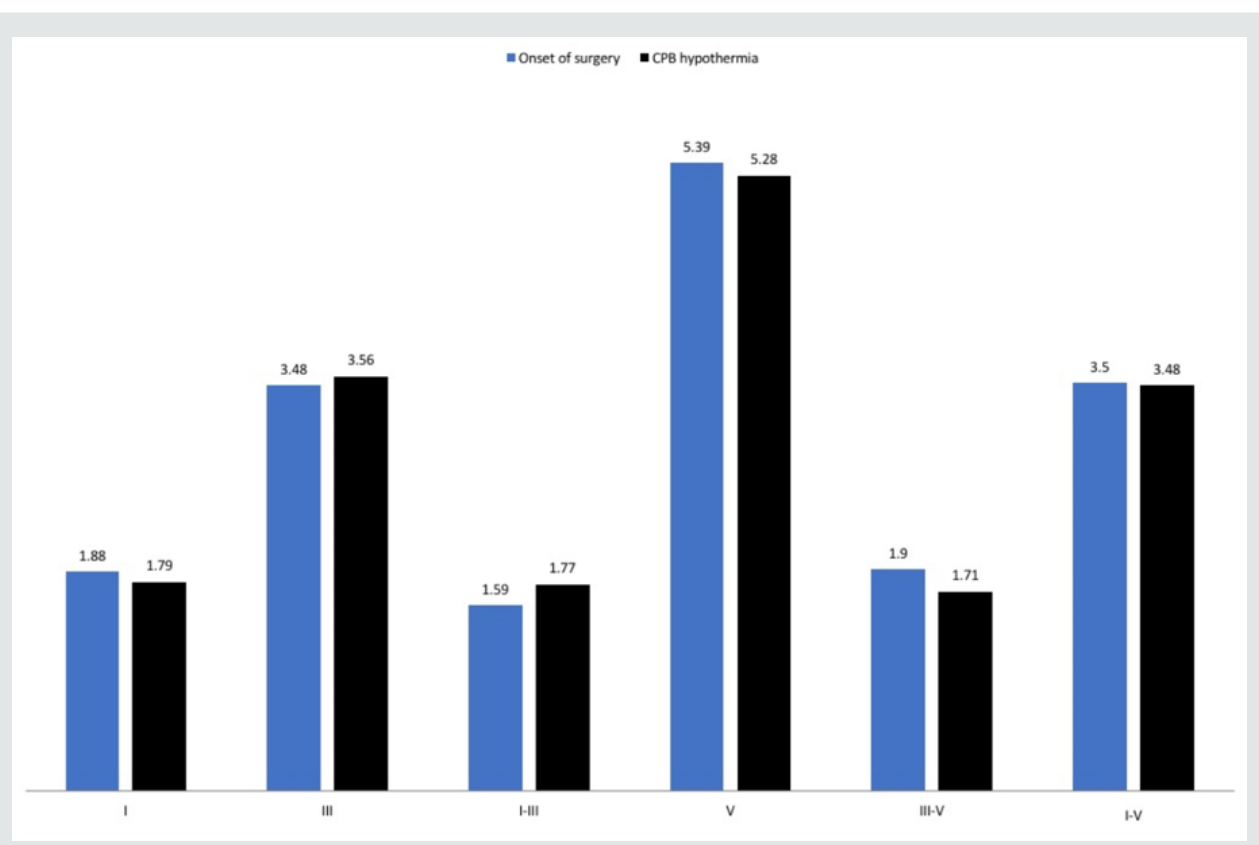

Figure 2. 


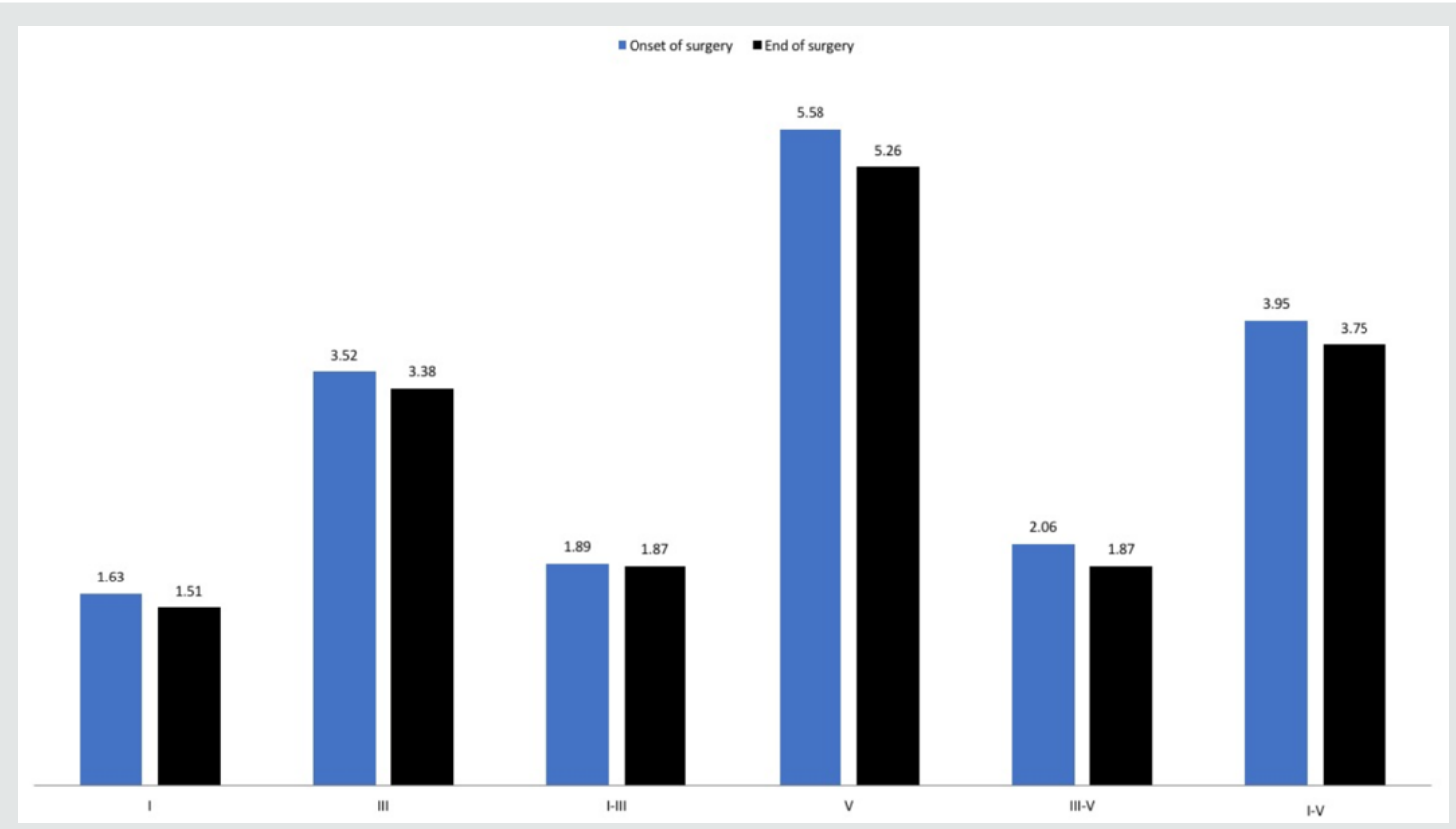

Figure 3.

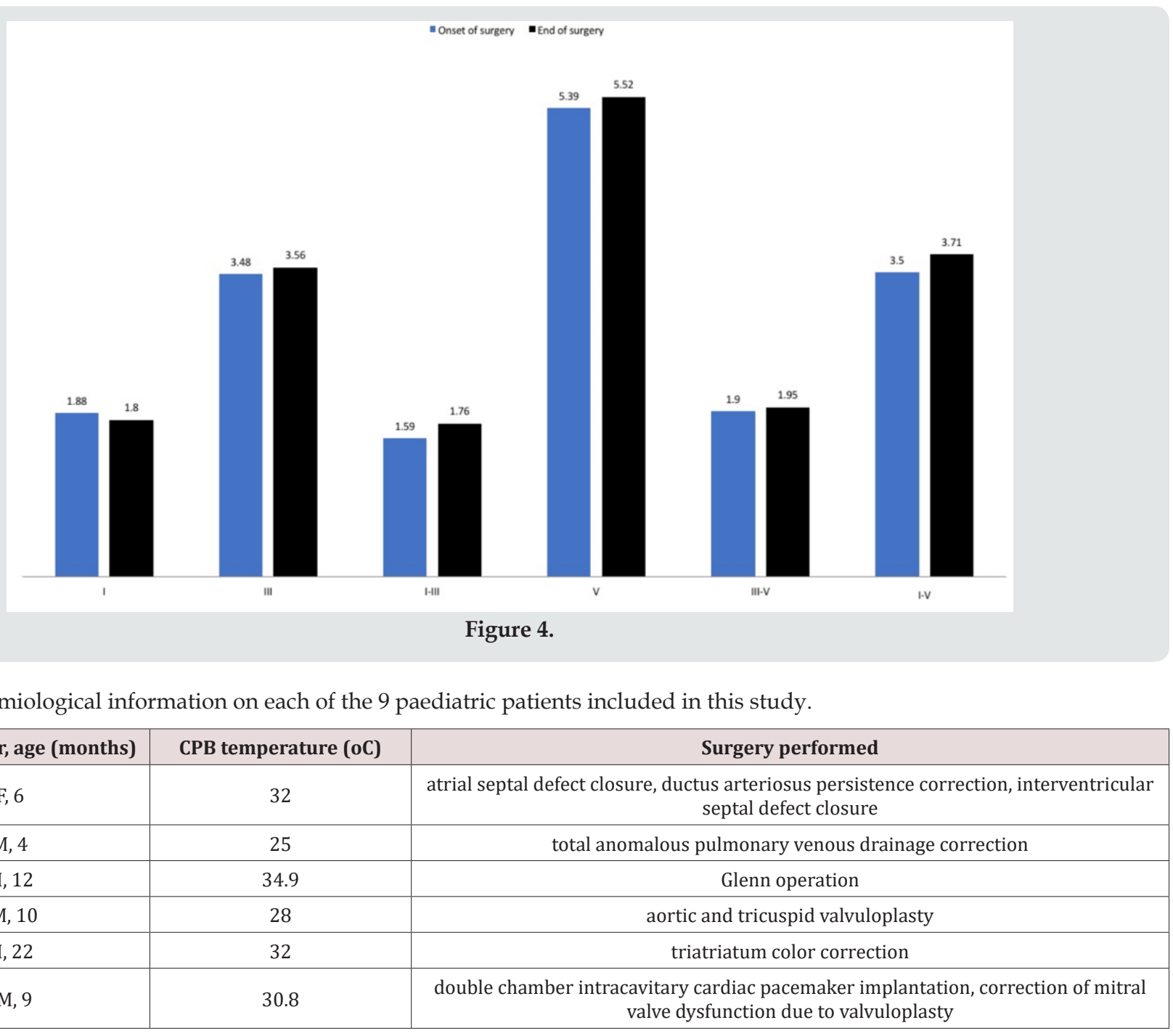




\begin{tabular}{|c|c|c|}
\hline $7, \mathrm{~F}, 12$ & 32.7 & interventricular septal defect closure \\
\hline 8, M, 28 days & 33 & bandaging \\
\hline $9, \mathrm{~F}, 22$ & 34.7 & atrial septal defect closure \\
\hline
\end{tabular}

*Down syndrome. Legend: $\mathrm{CPB}=$ cardiopulmonary bypass, $\mathrm{F}=$ female, $\mathrm{M}=$ male, oC = degrees Celsius.

Table 2: Mean latencies and mean interpeak latencies among each subject.

\begin{tabular}{|c|c|c|c|c|c|c|c|}
\hline \multirow{2}{*}{ Patient } & \multirow{2}{*}{ Side } & \multicolumn{3}{|c|}{ Latencies (ms) } & \multicolumn{3}{|c|}{ Interpeak latencies (ms) } \\
\hline & & I & III & $\mathbf{v}$ & I-III & $\mathbf{I}-\mathbf{V}$ & III-V \\
\hline \multirow{2}{*}{1} & $\mathrm{R}$ & 1.62 & 3.1 & 5.34 & 1.48 & 3.72 & 2.24 \\
\hline & $\mathrm{L}$ & 1.39 & 3.56 & 5.63 & 2.17 & 4.24 & 2.07 \\
\hline \multirow{2}{*}{2} & $\mathrm{R}$ & 1.52 & 3.33 & 5.28 & 1.81 & 3.76 & 1.95 \\
\hline & $\mathrm{L}$ & 1.71 & 3.51 & 5.31 & 1.8 & 3.6 & 1.8 \\
\hline \multirow{2}{*}{3} & $\mathrm{R}$ & 1.38 & 3.32 & 5.42 & 1.94 & 4.04 & 2.1 \\
\hline & $\mathrm{L}$ & 1.35 & 3.31 & 5.3 & 1.96 & 3.95 & 1.99 \\
\hline \multirow{2}{*}{4} & $\mathrm{R}$ & 1.69 & 3.71 & 5.55 & 2.02 & 3.86 & 1.84 \\
\hline & $\mathrm{L}$ & 1.66 & 3.42 & 5.27 & 1.76 & 3.61 & 1.85 \\
\hline \multirow{2}{*}{5} & $\mathrm{R}$ & 1.48 & 3.11 & 4.66 & 1.63 & 3.18 & 1.55 \\
\hline & $\mathrm{L}$ & 1.4 & 3.64 & 5.29 & 2.24 & 3.89 & 1.65 \\
\hline \multirow{2}{*}{6} & $\mathrm{R}$ & - & - & - & - & - & - \\
\hline & $\mathrm{L}$ & 1.32 & 3.41 & 5.32 & 2.09 & 4.00 & 1.91 \\
\hline \multirow{2}{*}{7} & $\mathrm{R}$ & 2.42 & 3.71 & 5.42 & 1.29 & 3.00 & 1.71 \\
\hline & $\mathrm{L}$ & 1.5 & 3.15 & 4.97 & 1.6 & 3.42 & 1.82 \\
\hline \multirow{2}{*}{8} & $\mathrm{R}$ & 2.16 & 3.87 & 5.67 & 1.71 & 3.51 & 1.79 \\
\hline & $\mathrm{L}$ & 1.93 & 3.73 & 6.03 & 1.8 & 4.1 & 2.3 \\
\hline \multirow{2}{*}{9} & $\mathrm{R}$ & 2.29 & 4.11 & 5.65 & 1.82 & 3.36 & 1.53 \\
\hline & $\mathrm{L}$ & 2.21 & 3.57 & 5.24 & 1.36 & 3.03 & 1.67 \\
\hline
\end{tabular}

Legend: $\mathrm{L}=$ left, $\mathrm{ms}=$ milliseconds, $\mathrm{R}=$ right

\section{Discussion}

There are few studies on the literature addressing the use of ABR to control brain stem activity during complex cardiac surgeries in children. The few studies available are conducted with small patient samples, given the complexities of the heart surgery itself. These studies show disappearance of the ABR waves between $180 \mathrm{C}$ and $260 \mathrm{C}$ and our study has observed that the $260 \mathrm{C}$ temperature may be a safe temperature to maintain central nervous system (CNS) integrity. However, later cognitive tests in this population would be necessary to confirm this hypothesis. When a subject is submitted to extracorporeal bypass and hypothermia, the physio pathologic alterations that occur in the central nervous system cause the waves I, III, and V to disappear in ABR. The literature reports that at body temperature of $260 \mathrm{C}$, waves I, III and V may disappear, and during hypothermia of $180 \mathrm{C}$, an isoelectric wave pattern is observed [10]. Unlike what we found in one of our patients (Subject 2) who maintained identifiable ABR waves even at a $25 \mathrm{oC}$ hypothermia temperature. In the present study, considering the number of patients evaluated at the moment of CPB and hypothermia, we observed that in only one subject the waves I, III, and $V$ disappeared, returning in the end of the surgery (Subject 6). As for the other subjects, ABR responses did not disappear, which does not agree with the literature and, therefore, does not indicate alteration in the auditory pathways of the patients submitted to these specific conditions of hypothermia, with temperatures of $25^{\circ} \mathrm{C}$.

Rodriguez et al. [11] also examined the effects of temperature changes on ABR in infants during hypothermic cardiopulmonary bypass. They found a significantly increasing in ABR latencies and a significantly decreasing in amplitude waves with cooling. Kusakari et al. [12] observed prolonged III and V waves among 10 children who underwent open heart surgery under hypothermia.

It was suggested that the prolonged latency of waves III and V was due to the slowed synaptic transmission in CNS and also due to the effects of the drugs used in general anaesthesia. Same findings were seemed by Hett et al. [13]. They recorded ABR potentials among 10 adults during $\mathrm{CPB}$ and hypothermia. Reduction in core temperature to $25 \mathrm{oC}$ resulted in an increase in latency and amplitude of the ABR; below this temperature the amplitude decreased but latency continued to increase until the ABR waves became completely flat between 21oC and 19oC. Kaga et al. [14] also observed prolonged ABR latencies among children with congenital heart disease undergoing cardiac surgery and with decreased body temperature. Yet, all waves disappeared below $25 \mathrm{oC}$. Interestingly, in all these 
previous studies, ABR was performed before general anaesthesia and throughout the surgery. In our study, the first register of ABR occurred soon after the anaesthetic sedation and it was possible to obtain the registers from waves I, III, and V, with adequate pattern, similar to what is expected for an individual with normal hearing, the scores of absolute latency, as well as the scores of the interpeak latency, of the ABR are similar to the normality range for new-borns and children up to 18 months old, as described in the literature. This fact may represent that general anaesthesia do not resound in the ABR acquisition, in agreement with literature findings. The $\mathrm{ABR}$ is resistant to alterations induced by anaesthetic drugs or alterations of consciousness [9]. Regarding the conditions related to CNS myelination, it is important to highlight the differences in the age groups of the subjects included in the present study.

Casali and Santos carried a study with 36 full-term and 30 premature infants by ABR testing.15 No gender differences were found, but significant difference in wave I, III and $\mathrm{V}$ absolute latencies at $80 \mathrm{~dB}$ and in wave $\mathrm{V}$ at $60 \mathrm{~dB}$ and $20 \mathrm{~dB}$ were observed in a comparison of absolute and interpeak latencies between fullterm and premature infants. An inverse correlation was found between age and absolute latencies, i.e. the older was the child, and the shorter was the latency [15]. Melo et al. [16] assessed 30 premature and 66 full-term infants by means of ABR waves. They found higher amplitude values in the preterm group among females, and no statistically significant difference related to latency. Changes in the latencies of waves I, III, and V were observed in the present study as well as its interpeak intervals in both ears in the different moments of the cardiac surgery, however these changes were not statistically significant, which does not suggest brain damage and does not agree with the studies found in relevant literature, which associates the ABR changes, as well as brain ischemia factors during cardiac surgery as possible causes of neurocognitive alterations, as it is expected for this example. Considering the sample and the conditions there in studied, the ABR did not detect auditory pathway injury as low as $250 \mathrm{C}$ hypothermia and cardiopulmonary bypass.

\section{Limitations}

This study has several limitations. First, the sample was relatively small. Second, the lack of uniformity in the age distribution of the patients. Unknown factors related to individual sensitivity for anaesthetic drugs or hypothermia. Lastly, the subjectivity to analyses the amplitude of ABR waves, made it difficult to interpret whether the register translated into brainstem function.

\section{Conclusion}

No changes were found in the absolute latencies and in the latency of the ABR intervals for situations of hypothermia and cardiopulmonary bypass.

\section{Conflicts of Interest}

The authors declare they have no potential conflicts of interest.

\section{References}

1. Rodriguez RA (2004) Human auditory evoked potentials in the assessment of brain function during major cardiovascular surgery. Semin Cardiothorac Vasc Anesth 8: 85-99.

2. Majnemer A, Limperopoulos C, Shevell M, Rosenblatt B, Rohlicek CV, et al. (2006) Long-term neuromotor outcome at school entry of infants with congenital heart defect requiring open heart surgery. J Pediatr 148: 72-77.

3. Limperopoulos C, Majnemer A, Shevell MI, Rohlicek C, Rosenblatt B, Tchervenkov C, et al. (2002) Predictors of developmental disabilities after open heart surgery in young children with congenital heart defects. J Pediatr 141: 51-58.

4. Bellinger DC, Jonas RA, Rappaport LR, Wypij D, Wernovsky G, et al. (1995) Developmental and neurologic status of children after heart surgery with hypothermic circulatory arrest or low-flow cardiopulmonary bypass. N Engl J Med 332: 549-555.

5. Limperopoulos C, Majnemer A, Shevell MI, Rosenblatt B, Rohlicek CV, et al. (2001) Functional limitation in young children with congenital heart defects after cardiac surgery. Pediatrics 108: 1325-1331.

6. Creighton DE, Robertson CM, Sauve RS, Moddermann DM, Alton GY, et al. (2007) Neurocognitive functional, and health outcomes at 5 years of age for children after complex cardiac surgery at 6 weeks of age or younger. Pediatrics 120: 478-486.

7. Anias CR, Lima MAMT, Kós OA (2004) Evaluation of the influence of age on auditory brainstem evoked potential. Rev Bras Otorrinolaringol 70: 84-89.

8. Akin A, Esmaoglu A, Tosun Z, Gulcu N, Aydogan H, et al. (2005) Comparison of propofol with propofol-ketamine combination in pediatric patients undergoing auditory brainstem response testing. Int J Pediatr Otorhinolaryngol 69: 1541-1545.

9. Hata R, Matsumoto M, Matsuyama T, Yamamoto K, Hatakeyama T, et al. (1998) Brainstem auditory evoked potentials during brainstem ischemia and reperfusion in gerbils. Neuroscience 283: 201-213.

10. Sousa LCA, Piza MRT, Ferez M, Rodrigues LS, Ruiz DB,et al. (2003) The $\mathrm{ABR}$ as a tool in the evaluation of brainstem function in surgeries with deep hypothermia and total cardiac arrest. Rev Bras Otorrinolaringol 69: 664-670.

11. Rodriguez RA, Edmonds Jr HL, Auden SM, Austin EH $3^{\text {rd }}$ (1999) Auditory brainstem evoked responses and temperature monitoring during pediatric cardiopulmonary bypass. Can J Anesth 46: 832-839.

12. Kusakari J, Inamura N, Sakurai T, Kawamoto K (1984) Effect of hypothermia upon the electrocochleogram and auditory evoked brainstem response. Tohoku K Exp Med 143: 351-359.

13. Hett DA, Smith DC, Pilkington SN, Abbott TR (1995) Effect of temperature and cardiopulmonary bypass on the auditory evoked response. Br J Anaesth 75: 293-296

14. Kaga K, Takiguchi T, Myokai K, Shiode A (1979) Effects of deep hypothermia and circulatory arrest on the auditory brain stem responses. Arch Otorhinolaryngol 225: 199-205.

15. Casali RL, Santos MFC (2010) Auditory brainstem evoked response: Response patterns of full-term and premature infants. Braz J Otorhinolaryngol 76: 729-738.

16. Melo Â, Sleifer P, Rechia IC, Biaggio EPV (2016) Cortical auditory evoked potentials in full term and preterm neonates: Gender and risk factors for hearing impairment. Audiol Commun Res 21: 1645. 
This work is licensed under Creative Commons Attribution 4.0 License

To Submit Your Article Click Here: Submit Article

DOI: $10.32474 /$ SJ0.2020.04.000187

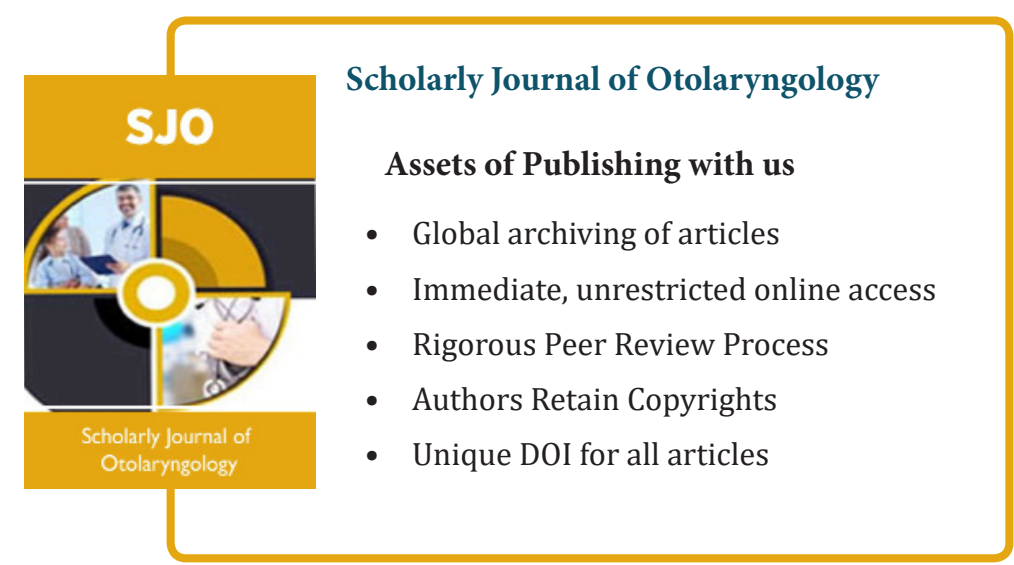

